\title{
Factores que potencian la autorregulación y el aprendizaje significativo en Primera Infancia
}

Volumen $6 \mathrm{~N} .{ }^{\circ} 48$ enero-junio de 2020

\author{
Enhancing Factors \\ of Self-regulation \\ and Meaningful \\ Learning in Early \\ Childhood
}

\author{
Fatores que melhoram \\ a autorregulação \\ e a aprendizagem \\ significativa na \\ primeira infância
}

Janeth Del Carmen Beltrán Espitia*

Elquin Eduar Mejía Loaiza**

Francisco Conejo Carrasco ${ }^{* * *}$

Fecha de recepción: 30-12-19

Fecha de aprobación: 26-05-20

\section{PARA CITAR ESTE ARTÍCULO}

Beltrán Espitia, J., Mejía Loaiza, E., Conejo Carrasco, F. (2020).

Factores que potencian la autorregulación y el aprendizaje significativo en Primera Infancia. Nodos y Nudos, 6(48). https://doi.org/10.17227/nyn.vol6.num48-11098.

Licenciatura en Pedagogia Infantil, Corporación Universitaria Minuto de Dios. jbeltranesp@uniminuto.edu.co

** Maestría en Educación, Corporación Universitaria Minuto de Dios. elkin.mejia@uniminuto.edu.co

** Maestría en Educación, Corporación Universitaria Minuto de Dios. francisco.conejo@uniminuto.edu.co 


\section{RESUMEN}

Este artículo presenta los resultados de una investigación que tuvo como objetivo conocer los factores que potencian la autorregulación del aprendizaje en los niños y las niñas de primera infancia, beneficiarios del proyecto "Pedagogía en contexto Aula Móvil", del Programa de Licenciatura en Pedagogía Infantil, de Uniminuto Soacha, Colombia; y la incidencia de dicha autorregulación en su aprendizaje significativo. Es menester subrayar que para este estudio se utilizó una metodología cualitativa con alcance exploratorio y descriptivo, logrado a través de una muestra no probabilistica intencional. Se encontró que la autorregulación y el aprendizaje significativo se potencian a través de: procesos motivacionales relacionados con la afectividad en el aula, el juego, la lúdica, el arte y los espacios para la libre expresión; procesos metacognitivos como los espacios para la autorreflexión, autovaloración, problematización de realidades, resolución de conflictos, experimentación y praxis; y con procesos de inteligencia emocional como el desarrollo de la confianza, la seguridad en si mismos y la autoestima.

Palabras clave: motivación; proceso cognitivo; aprendizaje activo; autodisciplina; primera infancia

\section{ABSTRACT}

This article presents the results of an investigation that aimed to know the factors that enhance the self-regulation of learning in early childhood boys and girls, beneficiaries of the project "Pedagogía en contexto Aula Móvil", of the Bachelor's Degree Program in Children's Pedagogy, from Uniminuto Soacha, Colombia; and the incidence of said self-regulation in their meaningful learning. It should be noted that this study used a qualitative methodology with an exploratory and descriptive scope, achieved through an intentional non-probabilistic sample. It was found that self-regulation and meaningful learning are enhanced through: motivational processes related to affectivity in the classroom, the act of playing, ludic, art, and spaces for free expression; with metacognitive processes such as spaces for self-reflection, self-assessment, problematization of realities, conflict resolution, experimentation, and praxis; and with emotional intelligence processes such as the development of confidence, self-confidence and self-esteem.

Keywords: motivation; cognitive process; active learning; self-discipline; early childhood

\section{RESUMO}

Este artigo apresenta os resultados de uma investigação que objetivou conhecer os fatores que potencializam a autorregulação da aprendizagem de meninos e meninas na primeira infância, beneficiários do projeto "Pedagogía en contexto Aula Móvil", do programa de Bacharelado em Pedagogia Infantil, da Uniminuto Soacha, Colômbia; e a incidência da referida autorregulação em sua aprendizagem significativa. É necessário ressaltar que para este estudo foi utilizada uma metodologia qualitativa com escopo exploratório e descritivo, obtida por meio de uma amostra não probabilistica intencional. Verificou-se que a autorregulação e a aprendizagem significativa são potencializadas por meio de: processos motivacionais relacionados à afetividade em sala de aula, o jogo, o lúdico, a arte e os espaços de livre expressão; com processos metacognitivos como espaços de autorreflexão, autoavaliação, problematização de realidades, resolução de conflitos, experimentação e práxis; e com processos de inteligência emocional, como o desenvolvimento da confiança, autoconfiança e autoestima.

Palavras-chave: motivação, processo cognitivo; aprendizagem ativa; autodisciplina; primeira infância 


\section{Introducción}

Con la firme intención de contribuir a los procesos de transformación social desde la educación, se hace necesario focalizar la mirada hacia la primera infancia, pues en esta etapa se configuran diferentes procesos de conocimiento que son muy importantes para el desarrollo integral de los sujetos. En esta mirada aparece la autorregulación del aprendizaje como una posibilidad para el fortalecimiento del aprendizaje significativo de los niños y niñas, algo que se viene trabajando en el proyecto "Pedagogía en contexto Aula Móvil", pero que vale la pena revisar y potencializar.

Soacha es el municipio más grande del departamento de Cundinamarca. En los últimos años es muy notorio el aumento de la población en esta localidad colombiana, a la que llegan familias de diferentes lugares del país, muchas huyendo del conflicto armado y otras que han padecido situaciones de pobreza extrema. La gran preocupación de todo esto es que los más afectados son muchos niños y niñas que ven amenazado su futuro, ya que llegan a una gran urbe en la que no hay mayores oportunidades para sus padres, ni de acceso a la educación para ellos. Es preciso decir que a través de la educación se va menguando la violencia y se van construyendo y consolidando territorios de paz.

Teniendo en cuenta la problemática subrayada anteriormente, surge en el municipio de Soacha el proyecto "Pedagogía en contexto Aula Móvil", creado por el programa de la Licenciatura en Pedagogía Infantil de Uniminuto, Centro Regional Soacha, cuya labor estriba en llevar la educación a los lugares más apartados y más vulnerables del sector. Los docentes en formación de este programa académico desarrollan estrategias innovadoras que permiten, a través de una educación no formal, brindar a estos niños y niñas un acercamiento importante al conocimiento.

Todas las acciones pedagógicas que se dan en el marco de este proyecto "Pedagogía en contexto Aula Móvil", están encaminadas al aprendizaje significativo de estos niños y niñas, fundamentadas principalmente en el juego, el arte, la cultura y, de manera muy especial, en la autorregulación del aprendizaje. Esta autorregulación hace referencia a procesos que los educandos logran por sí mismos y de forma proactiva, como afirma Zimmerman (2001) "el aprendizaje autorregulado no es algo que sucede a los estudiantes, sino que es algo que sucede por los estudiantes" (p. 33). En el caso particular de la población de estudio, se hace una apuesta pedagógica para que, desde muy temprano, estos niños y niñas tomen control de su aprendizaje y lleven a cabo estrategias metacognitivas tácticas, conscientes, reflexivas y de monitoreo permanente.

Esto lleva a pensar que en el potenciamiento de los procesos de autorregulación del aprendizaje se da una posibilidad significativa para que todos estos niños y niñas, que están en procesos de educación no formal, asuman un rol mucho más activo y autocontrolado de su aprendizaje. Por ello, es necesario ahondar en la búsqueda de elementos que, en el marco de la autorregulación del aprendizaje, permitan innovaciones pedagógicas que favorezcan de manera significativa el aprendizaje de estos niños y niñas de primera infancia. Tal propósito conduce a explorar la base de los factores que potencian la autorregulación de su aprendizaje.

Así, pues, es pertinente traer a colación temas tan relevantes como la motivación, la metacognición y la inteligencia emocional, los cuales se constituyen como elementos fundamentales para la autorregulación del aprendizaje. En relación con la motivación, Pila (2012) afirma,

que es el lazo que lleva esa acción a satisfacer la necesidad. En este sentido, la motivación se convierte en un activador de la conducta humana. Los estados motivacionales, lo mismo que los actitudinales, se generan por efecto de un conjunto de factores o variables que interactúan. (p. 18)

En cuanto a la metacognición, es importante decir que el desarrollo de la competencia metacognitiva se inmiscuye con todo el componente social, y es aqui donde adquiere relevancia en esta investigación, pues, como se ha subrayado con anterioridad, el contexto en el que se desenvolverá el estudio es netamente social. Es decir, que para llevar un aparataje teórico a la práctica es necesario primero conocer las necesidades y las respectivas problemáticas de las poblaciones con las que se está trabajando. Por un lado, es pertinente traer a colación a uno de los exponentes teóricos más relevantes: Flavell (1971), quien 
utilizó el término para hacer referencia a la meta memoria (González et ál., 2009).

Es preciso también retomar el postulado de Ardila, Diazgranados y Núñez (2009), quienes establecen que "la metacognición se describe en el nivel de conocimiento de los individuos sobre sus formas de entender el mundo y la capacidad de auto mejorar los procesos propios que concatenan la actividad gestiones y vinculación del conocimiento" (p. 29). Además, Ugartetxea (2001) afirma que la metacognición se aplica "en contextos objetivos, donde la relación enseñanza aprendizaje hace necesario pensar de manera simultánea en la articulación entre educación y motivación" (p. 32). Ahora bien, si Flavell (1976) se refiere al concepto como lo que se entiende de los propios procesos de aprendizaje o cognición, Carretero (2001) postula que se trata del conocimiento que se obtiene de la consciencia sobre los procesos cognitivos.

Por su parte, la inteligencia emocional es una habilidad que permite reconocer e intervenir en los estados de ánimo, siendo una etapa de predisposición por algún tipo de acción, es de suma importancia cuando se busca generar acciones de gran calidad. Sin el estado de ánimo propicio será inútil cualquier esfuerzo para mantener la calidad en las acciones. No obstante, el teórico Goleman (2011) afirma que la inteligencia emocional tiene que ver con: "habilidades tales como ser capaz de motivarse y persistir frente a las decepciones, controlar el impulso y demorar la gratificación, regular el humor y evitar que los trastornos disminuyan la capacidad de pensar, mostrar empatía y abrigar esperanzas" (p. 54). La cita anterior refiere al ámbito de la inteligencia emocional y a cómo esta hace parte objetiva de cada sujeto, la opción es trabajar de manera equitativa esta dimensión, pues en la contemporaneidad se ha visto cómo los lazos sociales hacen que se quiebre la comunicación y, por tanto, las relaciones interpersonales tienden a quebrarse; ello interviene de manera directa en el asunto de la inteligencia interpersonal.

Es pertinente, entonces, ahondar en el origen del concepto de inteligencia emocional: hacia 1990 dos brillantes pensadores, John Mayer y Peter Salovey, publican el artículo Emotional Intelligence. En su texto coinciden en que la inteligencia emocional es la capacidad para precisar de manera explícita las emociones y afectos, alternando la habilidad de autorregular y comprender todo aquello que de estas experiencias sensitivas se genera (Mayer y Salovey, 1997). De otro modo, para Pérez y Castejón (2006) es importante tener en cuenta la "teoría de las inteligencias múltiples" de Gardner, quien afirma que debe verse de forma biosociológica la inteligencia, él considera que es necesario el reconocimiento y el fortalecimiento de estas inteligencias (inteligencia interpersonal e inteligencia intrapersonal).

Por todo lo anterior, surge la pregunta de investigación: ¿Cuáles son los factores que potencian la autorregulación del aprendizaje en los niños y las niñas de primera infancia, beneficiarios del proyecto "Pedagogía en contexto Aula Móvil", del Programa de Licenciatura en Pedagogía Infantil de Uniminuto sede Soacha, y de qué manera incide dicha autorregulación en su aprendizaje significativo?

\section{Método}

En este apartado se presenta el diseño metodológico de la investigación, entendida la metodología desde la postura de Hernández (2010), como "un conjunto de procesos sistemáticos, críticos y empíricos que se aplican al estudio de un fenómeno" (p. 4). Esto permite ver que se trata de uno de los procesos más relevantes para determinar el nivel de confiabilidad de cualquier proceso investigativo. Para Ferreiro (2017), el método es un fin y la metodología un proceso de creación y cambio, ya que se concibe desde la idea de un maestro investigador, y es alli donde se integran estos dos elementos.

Se trató de una investigación cualitativa que, de acuerdo con Hernández et ál. (2010), se utiliza para comprender los sucesos en su naturaleza, sin intentar manipular los espacios ni controlar influencias de manera experimental, sino que se trata más bien de analizar la realidad subjetiva sin fundamentos estadisticos. En este sentido, se encontró en la metodología cualitativa una excelente posibilidad para acercarse a esa realidad subjetiva de estos niños y niñas de educación en la primera infancia, quienes integran la población de estudio, y poder identificar esos factores que potencian la motivación y el autocontrol en su aprendizaje. 
Este estudio tuvo un alcance exploratorio, entendido como aquel "que se efectúa sobre un tema u objeto desconocido o poco estudiado, por lo que sus resultados constituyen una visión aproximada de dicho objeto" (Arias, 2012, p. 23). Asimismo, este estudio tuvo un alcance descriptivo, que permitió interpretar la información recolectada para luego "comprender la descripción, el registro, el análisis, la interpretación y los procesos de los fenómenos" (Tamayo, 2003, p. 54). Esta investigación demandó también un trabajo hermenéutico interpretativo importante, en razón del tipo de población de estudio, ya que estuvo integrada por niños y niñas en edades muy tempranas, lo cual hacía necesario un proceso de observación sobre la cotidianidad de sus procesos de aprendizaje.

\section{Población}

La conforman los niños y las niñas beneficiarios del proyecto "Pedagogía en contexto Aula Móvil" de Uniminuto, sede Soacha, Colombia. Una población que demanda especial atención dadas sus condiciones de vulnerabilidad, y en las que una educación de calidad es fundamental para la trasformación de sus realidades sociales. La población estuvo representada por niños y niñas con unas condiciones de vulnerabilidad social, marcadas por situaciones de pobreza, violencia intrafamiliar y desplazamiento forzado. Para esta investigación se hace uso de la muestra no probabilística por conveniencia que, de acuerdo con lo expuesto por Goetz y LeCompte (1984), es aquella donde el investigador determina por adelantado un conjunto de atributos que deben poseer los sujetos de estudio, por lo tanto es una muestra intencionada, a conveniencia del investigador (Hernández et ál., 2010). Se determinó, entonces, una muestra representativa de ocho participantes en edades entre los cuatro y los seis años, considerando que son aquellos que representan más estabilidad y que han desarrollado procesos pedagógicos de manera más consistente. Su rango de edades permitió el despliegue de procesos pedagógicos mucho más activos, pues presentan mayor disposición a los diferentes espacios pedagógicos, como es el caso de aquellos mediados por el juego, el arte y la lúdica, entre otros.

\section{Instrumentos}

Las técnicas de recolección de datos buscan en los estudios cualitativos, de acuerdo con Hernández (2010), la obtención de datos que se conviertan en información de situaciones en profundidad, ocurridas en ambientes naturales y cotidianos de los participantes. Para este estudio se aplicó una entrevista semiestructurada y observación no participante. La entrevista semiestructurada es un instrumento que, según Mayan (2001), genera la oportunidad para comprender los puntos de vista de los participantes en la investigación, con respecto a los universos descritos por sus propias palabras. Admite, según este autor, la recolección de datos de los participantes a través de un conjunto de preguntas abiertas de manera ordenada, lo cual permite su saturación.

La técnica de la observación para Hernández et ál. (2010), en investigación cualitativa, tiene entre sus propósitos la exploración de los ambientes, y la descripción de la comunidad y actividades, como elementos más específicos que se pueden observar. Implica la puesta en acción de todos los sentidos, no es mera contemplación, lo cual significa adentrarse en la profundidad de las situaciones sociales, manteniendo un papel activo y de reflexión permanente del investigador; es estar atento a los sucesos, eventos e intenciones. De acuerdo con Giroux y Tremblay (2004), la observación no participante es aquella en la que el observador no participa en las actividades del grupo estudiado, así se conoce lo que sucede en el aula de clase, pero el investigador no interviene en las actividades que se realizan en el ambiente, de esta forma se reduce el sesgo de los datos e información que se logran. Teniendo en cuenta lo anterior, se diseñó una rejilla de observación a estudiantes, con indicadores orientados a identificar los procesos de motivación por el aprendizaje, metacognición e inteligencia emocional, como elementos fundamentales de autorregulación de su aprendizaje.

\section{Procedimiento metodológico}

El proceso metodológico en esta investigación se desarrolló en seis fases: diseño de instrumentos, validación de instrumentos, planificación, aplicación, sistematización y análisis de la información. Cada 
una de estas fases fue determinante para lograr un proceso objetivo y eficaz en relación con la calidad y confiabilidad de la información obtenida. Todas ellas contribuyeron de manera importante tanto en la etapa de preparación de la implementación y el trabajo de campo, como en la sistematización y análisis de la información.

La información recogida mediante los instrumentos (entrevista semiestructurada a docentes y observación a estudiantes) fue codificada en una matriz de análisis categorial, la cual se diseñó de tal manera que, con la información recolectada, primeramente, se alimentaran las subcategorías de análisis, y, por ende, se escalaran los datos a las categorías principales. Esta matriz se diseñó por medio de Excel, y permitió consignar los datos e identificar las recurrencias en las respuestas de los docentes y en los indicadores que se plantearon en la rejilla de observación. Cada vez que aparecía una coincidencia en los datos se procedía a asignar un color para ir agrupándolos, teniendo en cuenta su coincidencia (análisis de recurrencias). Aunque esta investigación siguió una metodología cualitativa, se hizo uso de procesos matemáticos básicos para establecer el nivel jerárquico; todo apoyado en la relación entre el número de coincidencias y el total de respuestas en la entrevista o indicadores en la observación.

Este procedimiento metodológico de análisis permitió un proceso de clasificación, categorización, organización en jerarquía y triangulación de los datos, hasta el punto de determinar los cinco hallazgos más importantes para cada una de las subcategorías de análisis (por instrumento). Tal información se procesó posteriormente a través de una matriz de triangulación para cada una de las subcategorías. Todo esto permitió exponer los datos a través de texto narrativo, tablas y diagramas descriptivos.

\section{Resultados}

A continuación, se presentan los resultados por categoría de investigación. Las tablas 1, 2 y 3 resumen los hallazgos correspondientes:

\section{Procesos de autorregulación del aprendizaje}

Tabla 1. Procesos de autorregulación del aprendizaje

\begin{tabular}{|c|c|}
\hline $\begin{array}{l}\text { Procesos } \\
\text { motivacionales }\end{array}$ & $\begin{array}{l}\text { Desarrollo de habilidades comunicativas y sociales para despertar el interés por el aprendizaje } \\
\text { La afectividad en el aula como elemento motivacional } \\
\text { El arte como fuente motivacional } \\
\text { Desarrollo de actividades lúdicas, recreativas y deportivas para despertar el interés por el aprendizaje } \\
\text { Desarrollo de actividades de encuentro directo con el entorno, como estrategia motivacional }\end{array}$ \\
\hline $\begin{array}{l}\text { Procesos } \\
\text { metacognitivos }\end{array}$ & $\begin{array}{l}\text { Espacios para la autorreflexión } \\
\text { Reconocimiento de pre-saberes } \\
\text { Espacios para la resolución de conflictos } \\
\text { Experimentación científica con elementos caseros } \\
\text { Desarrollo del aprendizaje activo a través de la experimentación }\end{array}$ \\
\hline $\begin{array}{l}\text { Procesos de } \\
\text { inteligencia } \\
\text { emocional }\end{array}$ & $\begin{array}{l}\text { Reconocimiento de la importancia de vida propia y del otro } \\
\text { Desarrollo de habilidades socio afectivas } \\
\text { Espacio para el autoconocimiento } \\
\text { Desarrollo de actividades en las que los niños y las niñas reconocen que son importantes para otros } \\
\text { Espacios para el desarrollo de la seguridad y confianza en sí mismo }\end{array}$ \\
\hline
\end{tabular}

Nota. Los procesos de autorregulación del aprendizaje se abordaron, desde la perspectiva motivacional, metacognitiva y de la inteligencia emocional. Fuente: elaboración propia. 
En cuanto a los procesos motivacionales en los diferentes espacios en los que se lleva a cabo la enseñanza-aprendizaje de estos niños y niñas, se destacan los siguientes: la afectividad de los actores que participan de la escena educativa, en especial por parte de los docentes y expresada en múltiples lenguajes, junto con las acciones, ambientes y procesos pedagógicos. También, el juego y la lúdica como elementos de mediación pedagógica que le permiten a estos niños y niñas aprender en medio de la realidad y la fantasía, y así desarrollar su capacidad de interacción y relación con otros.

Se notó el reto constante de innovación por parte de los docentes, trascendiendo de las aulas a espacios que permitan un mayor reconocimiento en interacción con el entorno real. Se hace muy evidente que el arte es uno de los elementos motivacionales más importantes cuando se trabaja con población de primera infancia, pues en estos niños y niñas se identificó un mayor nivel de motivación cuando tienen la posibilidad de exponer sus talentos en el canto, la música, el teatro, entre muchas otras expresiones, algo que se relaciona mucho con esa necesidad, por parte de los niños, del reconocimiento y valoración de los maestros y de sus compañeros de clase y que, entre otras cosas, alimenta de manera significativa su autoestima.

En cuanto a los procesos metacognitivos, se apreció desde la observación y desde el aporte de los docentes, que con estos niños y niñas se trabaja bastante en los procesos de autorreflexión, autovaloración, problematización de realidades, resolución de conflictos, experimentación y praxis. En los distintos espacios y procesos pedagógicos fue notoria la preocupación por ofrecer ambientes interactivos y llevar a cabo todo tipo de actividades y estrategias de aula para contribuir al desarrollo de su sentido táctico, estratégico, consciente y autovalorativo, elementos esenciales para que autorregulen su aprendizaje. Todo esto muy en relación con lo que expresa Carretero (2001), para quien la metacognición es el conocimiento que se obtiene de la consciencia sobre los procesos cognitivos.

Sobre los procesos de inteligencia emocional que se destacan en la enseñanza-aprendizaje de estos niños y niñas de primera infancia, es de notar un trabajo esforzado por parte de los docentes para orientar su práctica pedagógica a que tengan un mejor desarrollo multidimensional (corporal, socio afectivo, espiritual y de relación armónica con el entorno). Se da un lugar importante en cada uno de los espacios, actividades y procesos de aula al impulso de la confianza y seguridad en sí mismos, el autoconocimiento, el reconocimiento de su propio yo, el desarrollo de la autoestima y el sentido de vida. Así se identificó, por ejemplo, en los espacios para la exaltación de los talentos artísticos. Es de destacar que los docentes, como bien lo expresa Goleman (2006), alinean sus estrategias pedagógicas hacia la experiencia, donde se hace necesario pensar en sus pre-saberes sociales, culturales y otros.

\section{Prácticas pedagógicas y aprendizaje significativo}

Tabla 2. Prácticas pedagógicas y aprendizaje significativo

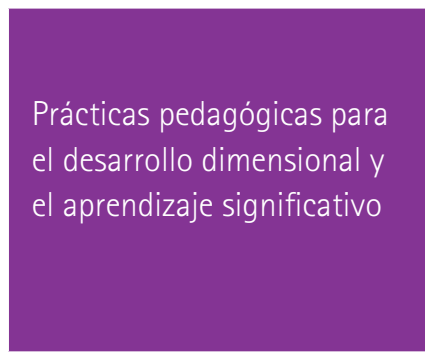

Desarrollo corporal kinestésico: movimiento, expresión y creatividad

Actividades para el desarrollo socio afectivo: juego y emocionalidad

Expresión corporal, movimiento y emociones

Desarrollo de las dimensiones estética y espiritual: arte, comunicación y sensibilidad

Interacción con el entorno para el fortalecimiento de la dimensión socio-política: historia, cultura y territorio 


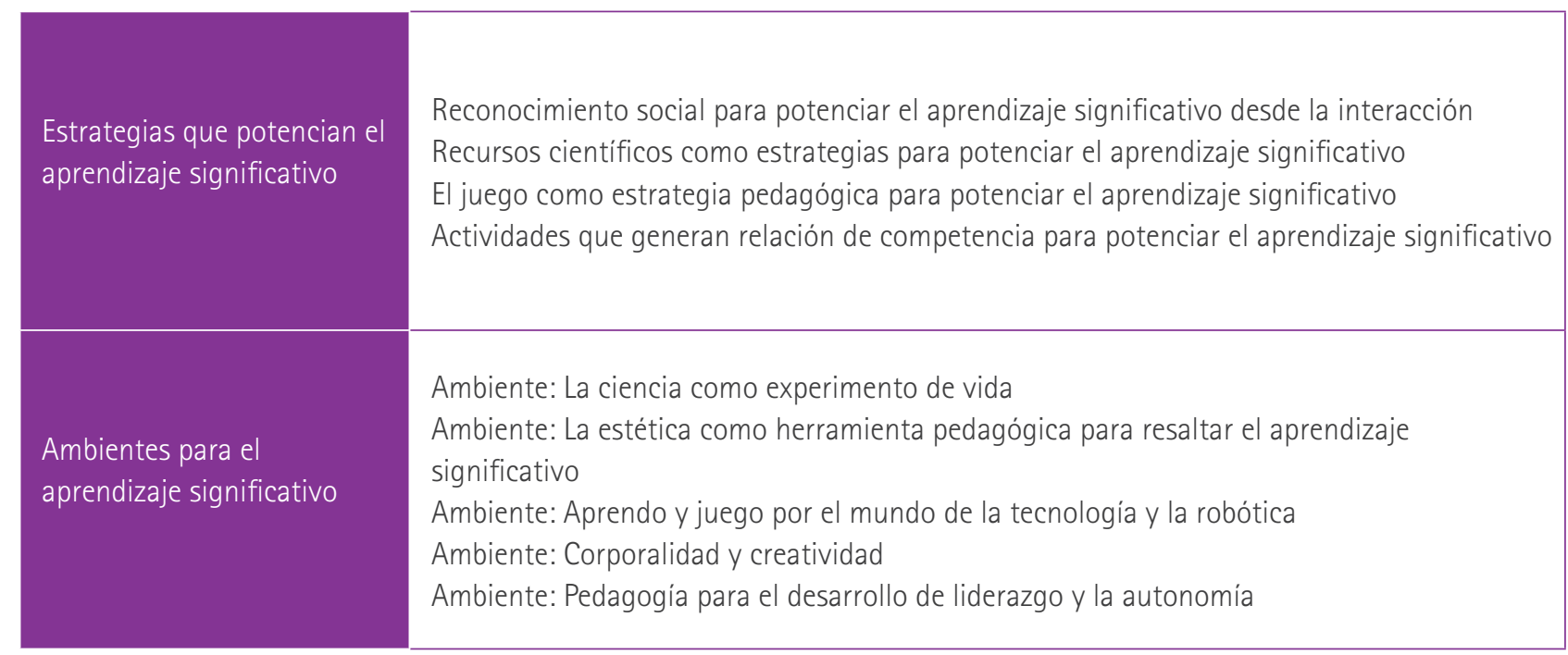

Nota. Se trabajaron las prácticas pedagógicas para el desarrollo dimensional y el aprendizaje significativo, así como las estrategias y ambientes para potenciar dicho aprendizaje. Fuente: elaboración propia.

En relación con el objetivo específico de Describir las prácticas pedagógicas aplicadas por los docentes en formación del Programa de Licenciatura en Pedagogía Infantil, de Uniminuto Regional Cundinamarca, sede Soacha, y que favorecen de manera importante el aprendizaje significativo en estos niños y niñas de primera infancia, se encontró que se está realizando un gran trabajo por parte de los docentes que orientan la enseñanza-aprendizaje de estos niños y niñas hacia el desarrollo de sus dimensiones humanas. Se obtuvo que se aplican actividades para el desarrollo corporal kinestésico, que trabaja especialmente movimiento, expresión y creatividad; desarrollo socio afectivo, con base en el juego y la emocionalidad; desarrollo estético y espiritual, apoyado especialmente en el arte, la comunicación y la sensibilidad; y desarrollo socio político, en el que se trabaja la interacción con el entorno a través de la historia, la cultura y el territorio.

Por otra parte, los resultados de la observación y la entrevista arrojaron que se llevan a cabo una serie de estrategias pedagógicas orientadas a potenciar los procesos de socialización, interacción, relación y comunicación asertiva a través del juego y la lúdica, también para el despliegue de competencias de exploración, experimentación e interacción con dispositivos tecnológicos. Además, se proveen espacios y recursos pedagógicos para el trabajo colaborativo.
Todas estas estrategias encuentran lugar en una serie de ambientes de aprendizaje con escenografías que permiten recrear espacios interactivos, entre estos: La ciencia como experimento de vida, La estética como herramienta pedagógica para resaltar el aprendizaje significativo, Aprendo y juego por el mundo de la tecnología y la robótica, Corporalidad y creatividad y Pedagogía para el desarrollo de liderazgo y la autonomía. Todas esas actividades, estrategias y ambientes pedagógicos, según los resultados obtenidos, son determinantes para el aprendizaje significativo. Todo esto está muy en relación con lo que afirma Zabala (2002): "las prácticas pedagógicas como un conjunto amplio de procedimientos, acciones y estrategias que permite regular las intenciones en los espacios educativos, por medio de lo que el maestro enseña, ayuda a construir y darle significados" (p. 35).

En efecto, las prácticas pedagógicas constituyeron un sistema de interacciones que en este caso se dio en un escenario diferente al aula de clase, reconociendo el juego como pieza clave en el proceso de aprendizaje, los espacios educativos son ambientes de aprendizaje que fortalecieron las competencias a través de la ciencia como experimento de vida, la interactividad con aparatos de la tecnología y la robótica, como base de competencia que se refiere al desarrollo multidimensional y al aprendizaje significativo. 


\section{Factores de mayor influencia en la autorregulación del aprendizaje}

Tabla 3. Factores de mayor influencia en la autorregulación del aprendizaje

\begin{tabular}{|c|c|}
\hline $\begin{array}{l}\text { Factores de mayor } \\
\text { influencia en la } \\
\text { motivación por el } \\
\text { aprendizaje }\end{array}$ & $\begin{array}{l}\text { El lenguaje del amor, un elemento esencial para la motivación por el aprendizaje } \\
\text { La motivación por el aprendizaje a través de las artes y la exaltación de los talentos } \\
\text { Interacción, relación y libertad de expresión, elementos fundamentales para la motivación por el } \\
\text { aprendizaje } \\
\text { El juego como herramienta lúdica pedagógica para la motivación por el aprendizaje } \\
\text { Interacción con el entorno, un factor determinante para la motivación por el aprendizaje }\end{array}$ \\
\hline $\begin{array}{l}\text { Factores de } \\
\text { mayor influencia } \\
\text { en el desarrollo } \\
\text { metacognitivo }\end{array}$ & $\begin{array}{l}\text { La autorreflexión permanente como estrategia metacognitiva } \\
\text { Aprendizaje en la base de los conocimientos previos, un factor importante para dar mayor sentido a } \\
\text { la construcción de nuevo conocimiento } \\
\text { Problematización de la realidad y participación activa en la resolución de conflictos, aspectos } \\
\text { esenciales para el desarrollo metacognitivo } \\
\text { La experimentación científica como aspecto fundamental para el desarrollo de la responsabilidad y la } \\
\text { autonomía en el aprendizaje } \\
\text { El aprendizaje activo a través de la praxis, un elemento esencial para el desarrollo metacognitivo }\end{array}$ \\
\hline $\begin{array}{l}\text { Factores de mayor } \\
\text { influencia en } \\
\text { el desarrollo de } \\
\text { la inteligencia } \\
\text { emocional }\end{array}$ & $\begin{array}{l}\text { Sentido de vida, un aspecto fundamental para la inteligencia emocional } \\
\text { Desarrollo de las competencias socio afectivas } \\
\text { El autoconocimiento un factor esencial para el autocontrol emocional } \\
\text { Desarrollo de la autoestima y el auto concepto } \\
\text { La autoconfianza, amor propio y espiritualidad }\end{array}$ \\
\hline
\end{tabular}

Nota. Se presentan los factores de mayor influencia en la motivación, metacognición, inteligencia emocional, aspectos fundamentales de la autorregulación del aprendizaje. Fuente: elaboración propia.

Sobre el objetivo especifico de Establecer los factores de mayor influencia en la autorregulación del aprendizaje en estos niños y niñas de primera infancia, se encontró que, desde una perspectiva motivacional, estos niños empiezan a mostrar procesos de autocontrol, autogestión y monitoreo de su aprendizaje, a través de elementos tan importantes como el lenguaje afectivo en todos y cada uno de los espacios y por parte de todos los seres que constituyen el acto educativo. El uso del juego y el arte no solo contribuye a los procesos de interacción y socialización, sino a cautivar su interés y atención, y a que puedan aprender con alegría y disfrute de cada proceso de aprendizaje.

Sumado a lo anterior, se resaltan los espacios para la libre expresión y para reconocer su importancia, para "dar lugar" a su ser, y que se sientan reconocidos, valorados y queridos. También, trascendiendo las barreras de las cuatro paredes del aula y llevándolos a ese encuentro con el entorno real, se logra activar su motivación y, a su vez, que tengan el impulso de tomar control de su propio proceso de aprendizaje.

La metacognición como elemento esencial de autorregulación del aprendizaje se potencia a través de actividades y espacios para la reflexión, ejercicios de aprendizaje en la base de los saberes previos, espacios para el reconocimiento y problematización de situaciones del entorno real y ejercicios interactivos para la práctica y la experimentación. También todo tipo de procesos que les permiten fortalecer su sentido táctico, estratégico, consciente, reflexivo y de capacidad de autonomía. 
Desde la perspectiva de la inteligencia emocional, se hizo evidente que el desarrollo y control emocional de estos niños y niñas se relacionan de manera muy directa con su entorno familiar, la integración de sus seres queridos a los espacios de aprendizaje es fundamental para determinar mayores niveles de confianza y seguridad en sí mismos, pues se sienten mucho más acompañados, protegidos y apoyados. Al incrementar sus niveles de seguridad y confianza en sí mismos, lo harán también con los seres que están en su entorno.

Se conoció que son importantes los espacios para la autovaloración, para el establecimiento de las relaciones socio afectivas con los pares, para la percepción positiva de sí mismos (autoestima, autoconcepto y autoimagen) y para el crecimiento personal y espiritual. Goleman (1995) considera que para desarrollar la inteligencia emocional es necesario conocer y manejar las propias emociones, motivarse a uno mismo, reconocer las emociones de los demás y establecer relaciones. En este sentido, la autorregulación incluye la percepción positiva de sí mismos y el crecimiento personal y espiritual.

\section{Discusión}

Luego de relacionar los objetivos específicos, es oportuno ahora, ofrecer respuesta a la gran pregunta de investigación: ¿Cuáles son los factores que potencian la autorregulación del aprendizaje en los niños y las niñas de primera infancia, beneficiarios del proyecto "Pedagogía en contexto Aula Móvil", del Programa de Licenciatura en Pedagogía Infantil de Uniminuto sede Soacha, y de qué manera incide dicha autorregulación en su aprendizaje significativo?

Después de un análisis riguroso de los datos obtenidos mediante la observación de los procesos de enseñanza-aprendizaje en estos niños y niñas beneficiarios del proyecto "Pedagogía en contexto Aula Móvil", y a través de la entrevista a sus docentes para conocer desde sus perspectivas los factores que potencian los procesos de autorregulación del aprendizaje en ellos, es preciso decir que, aunque se trata de niños y niñas de primera infancia, este estudio develó la importancia de trabajar desde edades tempranas la autorregulación del aprendizaje, para lo cual es fundamental el desarrollo de la capacidad metacognitiva, de inteligencia emocional $y$, de manera especial, la motivación por el aprendizaje. Teniendo esto presente, es fundamental reconocer que la autorregulación del aprendizaje en estos niños y niñas, desde estos tres pilares, se potencia de la siguiente manera:

En estos pequeños estudiantes la autorregulación se potencia a través de procesos motivacionales que se activan con elementos tan importantes como: afectividad en todos los espacios, ambientes y procesos pedagógicos, por parte de todos los actores en la escena educativa y expresada por medio de múltiples lenguajes; actividades, ambientes y estrategias de aprendizaje con mediación del juego y la lúdica; proveer espacios para socialización, relación e interacción, tanto al interior del aula como con el entorno real; proporcionar espacios para la libre expresión a través del arte; y estimular la autoestima por medio de frases de reconocimiento y exaltación de los talentos y capacidades. Todo esto se soporta en lo que propone Ugartetxea (2001), quien afirma que la metacognición se aplica "en contextos objetivos, donde la relación enseñanza aprendizaje hace necesario pensar de manera simultánea en la articulación entre educación y motivación" (p. 32).

También se estableció que la autorregulación en estos niños y niñas se potencia a través de procesos metacognitivos como: autorreflexión, autovaloración, problematización de realidades, resolución de conflictos, experimentación y praxis, y todo tipo de actividades y procesos pedagógicos para el desarrollo del sentido táctico, estratégico, consciente y autovalorativo. De otro modo, se notó que la autorregulación del aprendizaje en estos niños y niñas se favorece de modo relevante por medio del despliegue de procesos de inteligencia emocional, y que se dan a partir de estrategias de aula para que ellos reconozcan su ser multidimensional, es decir, de las dimensiones humanas, especialmente la afectiva, la social, la espiritual y la estética corporal; también el impulso de la confianza y seguridad en sí mismos, el autoconocimiento, el reconocimiento de su propio yo y el desarrollo de la autoestima y el sentido de vida. Al respecto, Goleman (2011) afirma que la inteligencia emocional tiene que ver con habilidades como: "ser capaz de motivarse y persistir frente a las 
decepciones, controlar el impulso y demorar la gratificación, regular el humor y evitar que los trastornos disminuyan la capacidad de pensar, mostrar empatía y abrigar esperanzas" (p. 54).

Todo esto implica que los actores que rodean los procesos de enseñanza-aprendizaje de estos niños y niñas son quienes proveen elementos para que ellos mismos logren activar el empoderamiento, la autogestión, el autocontrol, la autodirección y el monitoreo de su aprendizaje. Queda claro que son ellos mismos los que pueden autorregular su aprendizaje, por supuesto, entendiendo que están iniciando en su educación, pero que desde muy temprano pueden empezar procesos fundamentales, en este sentido que proyecten y estructuren su pensamiento hacia el logro de sus metas.

Se identificó que la motivación como elemento esencial de la autorregulación del aprendizaje se constituye en el impulso para disponerse a aprender y para conectarse de manera activa con todos los procesos de aula, lo que favorece el aprendizaje significativo. Se notó que cuando se llevan a cabo procesos tácticos, estratégicos, conscientes y reflexivos, es decir, procesos metacognitivos propios de autorregulación del aprendizaje, se potencia el aprendizaje significativo. También, que cuando se trabaja por el desarrollo de habilidades emocionales y socio-afectivas que les permitan estabilidad y armonía, pueden lograr más control y empoderamiento de su propio proceso de aprendizaje. Todo lo anterior reafirma la postura de Monereo (2001), para quien la autorregulación es la "facultad de tomar decisiones que permitan regular el propio aprendizaje para aproximarlo a una determinada meta, en el seno de unas condiciones específicas que forman el contexto de aprendizaje" (p. 12).

\section{Referencias}

Ardila, J., Diazgranados, Y. y Núñez, E., (2009) Habilidades metacognitivas y rendimiento académico en estudiantes de vI y vI semestre de los programas de ingeniería de sistemas e ingenieria industrial horario nocturno de la Universidad del Magdalena. http://repositorio.unimagdalena.edu.co/jspui/bitstream/123456789/674/1/ SP-00071
Arias, F. G. (2012). El proyecto de investigación. Episteme. shorturl.at/prvHM

Carretero, M. (2001). Metacognición y educación. Aique.

Ferreiro, R. (2017). ¿Cómo ser maestro investigador?: El Método JAVI - Vademécum. Corporación Universitaria Minuto de Dios.

Flavell, J. (1971). First discussant's comments: What is memory development the development of? Human Development, 14(4), 272-278. https://doi.org/10.1159/000271221

Flavell, J. H. (1976). Aspectos metacognitivos de la resolución de problemas. En L. B. Resnik (ed.). La naturaleza de la inteligencia (pp. 231-235). Erlbaum.

Giroux, S. y Tremblay, G. (2004). Metodologia de las ciencias humanas. Fondo de Cultura Económica. https:// imas2010.files.wordpress.com/2010/06/metodologiade-las-cchh-s-giroux-g-tremblay.pdf

Goetz, J. y Lecompte, M. (1984). Etnografía y diseño cualitativo en investigación educativa. Morata. https://www. academia.edu/11082069/Etnograf\%C3\%ADa_y_dise\%C3\%B10_cualitativo_en_investigaci\%C3\%B3n_educativa

Goleman, D. (1995). Inteligencia emocional. Kairós.

Goleman, D. (2006). Inteligencia social. Kairós.

Goleman, D. (2011). Liderazgo. El poder de la inteligencia emocional. Ama.

González, J., Mayor, J. y Suengas, A. (1995). Estrategias metacognitivas. Sintesis S. A. https:// upeldem.files.wordpress.com/2017/04/estrategias-metacognitivas-aprender-a-aprender-y-aprender-a-pensar-juan-mayor-aurora-suengas-y-javier-gonzc3a1lez-marquc3a9s-cap-4-y-5-1.pdf

Hernández, R. (2010). Metodología de la Investigación. McGraw-Hill.

Hernández, R., Fernández, C. y Baptista, P. (2010). Metodologia de la Investigación. McGraw-Hill.

Mayan, M. J. (2001). Una introducción a los métodos cualitativos. Qual Institute Press. https://sites.ualberta.ca/ iiqm/pdfs/introduccion.pdf

Mayer, J. y Salovey, P. (1997). Emotional Development and Emotional Intelligence. http://ei.yale.edu/journal/emotional-development-and-emotional-intelligence-educational-implications/

Monereo, C. (2001). La enseñanza estratégica, enseñar para la autonomía. Revista Aula de Innovación, 100. http:// www.quadernsdigitals.net/datos/hemeroteca/r_57/ nr_625/a_8481/8481.pdf 
Pérez, N. y Castejón, J. L. (2006). Relaciones entre la inteligencia emocional y cociente intelectual con el rendimiento académico en estudiantes universitarios. Revista electrónica de motivación y emoción, 9 (22). https://dialnet. unirioja.es/servlet/articulo?codigo $=2123905$

Pila, J. E. (2012). La motivación como estrategia de aprendizaje en el desarrollo de competencias comunicativas de los estudiantes (Tesis de Maestría). Universidad de Guayaquil. http://repositorio.ug.edu.ec/bitstream/redug/1659/1/tesis\%20completado\%20de\%20motivacion.pdf

Tamayo, M. (2003). El proceso de la investigación cientifica. Noriega Editores. https://es.scribd.com/doc/12235974/ Tamayo-y-Tamayo-Mario-El-Proceso-de-la-Investigacion-Cientifica
Ugartetxea, J. (2001). Motivación y Metacognición, más que una relación. Revista Electrónica de Investigación y EvaIuación Educativa, 7 (2), 51-71. https://www.uv.es/relieve/v7n2/RELIEVEv7n2_1.pdf

Zabala, A. (2002). La práctica educativa, cómo enseñar. Graó Zimmerman, B. J. (2001). Theories of Self-Regulated Learning and Academic Achievement: An Overview and Analysis. En B. J. Zimmerman y D. H. Schunk (Eds.), Self-regulated Learning and Academic Achievement: Theoretical Perspectives (pp. 1-39). https://www.researchgate.net/ publication/243775466_Self-Regulated_Learning_and_ Academic_Achievement_An_Overview

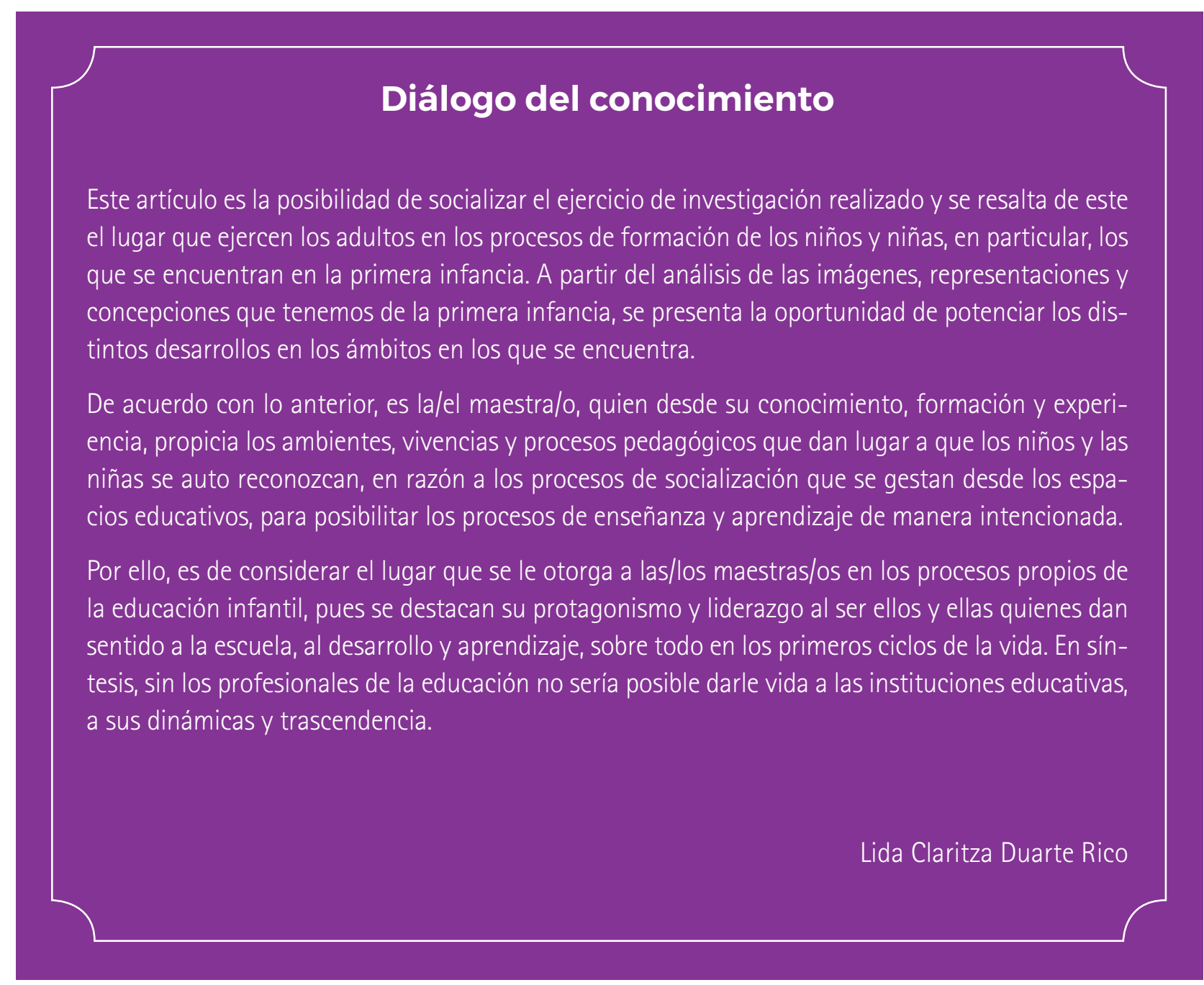


